\title{
Correction to: Antiretroviral treatment indications and adherence to the German-Austrian treatment initiation guidelines in the German ClinSurv HIV Cohort between 1999 and 2016
}

\author{
Melanie Stecher ${ }^{1,2}$ (D) Philipp Schommers ${ }^{1,2}$. Daniel Schmidt ${ }^{3,4}$. Christian Kollan ${ }^{3}$. \\ Barbara Gunsenheimer-Bartmeyer $^{3}$. Clara Lehmann ${ }^{1,2} \cdot$ Martin Platten $^{1} \cdot$ Gerd Fätkenheuer $^{1,2}$. \\ Jörg Janne Vehreschild ${ }^{1,2}$ • ClinSurv Study Group
}

Published online: 25 February 2019

(c) Springer-Verlag GmbH Germany, part of Springer Nature 2019

\section{Correction to: Infection https://doi.org/10.1007/s15010-018-1248-8}

In this article the cooperating partners of the ClinSurv HIV cohort were not listed in the acknowledgements. The correct paragraph appears below:

\section{Acknowledgements}

This study was funded by the German Center for Infection Research. Initial results of this study were presented at the '21st International Workshop on HIV and Hepatitis Observational Databases' in Lisbon, Portugal 2016, Abstract no: 109.

This research was significantly supported by the ClinSurv HIV Study Group. We thank all cooperating partners in the ClinSurv HIV cohort study centers:

Berlin: Dr. F. Bergmann, M. Warncke: Charité, Universitätsmedizin Berlin

The original article can be found online at https://doi.org/10.1007/ s15010-018-1248-8.

Melanie Stecher

melanie.stecher@uk-koeln.de

$\triangle$ Jörg Janne Vehreschild

joerg.vehreschild@uk-koeln.de

1 Department I for Internal Medicine, University Hospital of Cologne, Cologne, Germany

2 Partner Site Cologne-Bonn, German Center for Infection Research (DZIF), Cologne, Germany

3 Department of Infectious Disease Epidemiology, Robert Koch Institute, Berlin, Germany

4 Charité - University Medicine Berlin, Berlin, Germany
Bonn: Prof. Dr. J. Rockstroh, Dr. J. Wasmuth, S. Hass: Universitätsklinikum Bonn

Düsseldorf: Dr. B.-O. Jensen, C. Feind: Universitätsklinik Düsseldorf

Essen: Dr. S. Esser, P. Schenk-Westkamp: Universitätsklinikum Essen

Frankfurt: PD Dr. C. Stephan, Dr. A. Haberl, P. Schott: HIV Center Frankfurt

Hamburg: Prof. Dr. A. Plettenberg, Dr. T. Lorenzen, F. Kuhlendahl: ifi (Inst. für interdisziplinäre Medizin)

Drs. A. Adam, K. Schewe, S. Fenske, T. Buhk, Prof. H.J. Stellbrink, C. Hoffmann, D. Radzuweit, A. Mainka: ICH (Infektionsmedizinisches Centrum Hamburg)

Dr. O. Degen, G. Schäfer, M. Franz: Universitätsklinikum Hamburg Eppendorff

Hannover: Prof. Dr. M. Stoll, S. Gerschmann, R. Beider: Medizinsche Universität Hannover

Kiel: Prof. Dr. H. Horst, S. Trautmann: Universitätsklinikum Schleswig-Holstein

Köln: Prof. Dr. G. Fätkenheuer, Prof. Dr. J. Vehreschild, P. Schommers: Universitätsklinikum Köln

München: Prof. Dr. J. Bogner, B. Sonntag: Ludwig-Maximilians Universität München

Rostock: Dr. C. Fritzsche: Universitätsklinikum Rostock 